DISCUSSION:

Paper:

Author:

SHUTER:

WAYTE:

WANG:

WAYTE:

SOFUE:

WAYTE:

Paper:

Author:

MOULD:

IRWIN:

HUGHES:

IRWIN:

SCHOMMER:

IRWIN:

\section{THE LMC-SMC-GALAXY SYSTEM}

\author{
The interacting Magellanic System \\ S.R. Wayte
}

I would like to point out that in tidal models it is also possible to obtain constant radial velocity along the length of the Stream clouds MS I through MS VI, if each spins as it orbits so as to keep the same face towards the Galactic centre.

Yes, I concede that.

We do see somewhat enhanced $x$-ray emissivity along the suggested interacting front to the eastern side of the LMC. The x-ray emitting temperature is expected from the velocity $\sim 300 \mathrm{~km} / \mathrm{s}$ and the implied density of the $x$-ray emitting gas is $\sim 5 \times 10^{-4} \mathrm{~cm}^{-3}$.

That will be nice.

1. There are many HI clouds in the opposite side to the stream. The tidal model can well reproduce the feature. However, the ram-pressure model seems to have difficulty on this point.

2. Tidal tails are usually very thin, and need no confinement if the tail's age is less than a few $10^{8} \mathrm{yr}$. Note that the stream is the result of a rather recent encounter between $L M C$ and $S M C$ (debris from $S M C$ ): not due to interactions among $L M C+S M C+M i l k y w a y$ for 2 $x 10^{9}$ yrs.

1. The HVCs ahead of the Magellanic Clouds can be reproduced by tidal models - true. However, HVCs are ubiquitous and the HVCs ahead are not just all along the stream line. These HVCs fit nicely into a population of HVCs in the galactic halo which can be swept up by the Magellanic System. Tidal tails can be thin, yes. However, the Stream has bulk motions which if not confirmed will double the stream width in $\sim 5 \times 10^{7}$ years. Tidal 2. No. tails so far produced need a time of order $10^{9}$ years to form.
Optical surveys of the Magellanic Bridge, Stream and outer halos M.J. Irwin

What indicators of an intermediate age population did you lookfor, and fail to find, in the Bridge?

Red horizontal branch, AGB and red carbon stars. Its worth noting that the bridge region is only $\sim 3^{\circ}$ across; the outer halo populations of the Clouds almost overlap.

Is there any evidence of a tilt in the disk of the LMC from the horizontal branches of the outer LMC halo fields?

We do not yet have adequate photometric calibration for these outer halo fields but given the well defined position of the HB in blue photographic CMDs it would certainly be possible to investigate the tilt this way.

Do any of these outer clusters show up as increments in your counts? Do you find additional clusters?

All of the known outer clusters show up as increments in the counts. However, the difficulties in finding additional sparse (faint) clusters this way, is the large contamination 
of the counts by both background galaxy clusters and image fragmentation of bnynt objects, leading to considerable visual effort being required.

TOBIN:

IRWIN:

Paper:

Author:

PETTINI:

FUJIMOTO:

WAYTE:

FUJIMOTO:

VAN DEN BERGH:

FUJIMOTO:

Paper:

Author:

LANEY:

FEAST:
You say the blue bridge stars have ages $\sim 10^{8}$ yr. How much less? The colour magnitude diagram you published in Nature shows objects with early B colours, which surely must be much younger than $10^{8}$ yr?

Isochrone fitting to CCD CMD's of the associations of bridge stars reveal an age spread between 50 and 100 million years. One problem with directly interpreting the bright end of the blue main sequence is contamination by Galactic $\mathrm{HB}$ stars, hot $\mathrm{F}$ subdwarfs and white dwarfs. These associations are so large $(50 \mathrm{pc}-1 \mathrm{kpc})$ that there is always some contamination from the foreground stars, which can only be eliminated using radial velocity information.

Asymmetric distribution of gas in the LMC and dynamical condition for globular cluster formation

Mitsuaki Fujimoto, Yasuki Kumai

There are two clusters in the Galactic halo (Pol 12 and NGC 362) which seem to be a few Gyr younger than all the other Galactic globulars. Do you think it possible that they actually formed within the Magellanic Clouds, and were later stripped from them at a close perigalactic passage?

I don't think that these globular clusters came from the Magellanic Clouds. The perigalactic center of the Magellanic Clouds was far more than $50 \mathrm{Kpc}$ at that time. These globulars could be formed from primordial gas clouds, infalling with velocity of more than $100 \mathrm{~km} / \mathrm{s}$ and compressed by collision with Galactic halo gas.

If the SMC passed through the gaseous $L M C$, i.e. within $5 \mathrm{kpc}$, then should not the Spitzer-Baade process sweep out much SMC gas? So why is the SMC still so gaseous? Indeed, more gaseous than expected for the SMC's morphological type.

Compression to southwest of SMC. Some swept out.

If cloud collisions with relative velocities of $50-100 \mathrm{~km} / \mathrm{s}$ are required to form globular clusters, how do you account for the existence of 5 globulars in the Fornax dwarf spheroidal galaxy?

If the Fornax dwarf galaxy is a fragment of a more massive original, 5 globulars you pointed out could be generated from primordial gas clouds compressed by primordial turbulence in this object before fragmentation. If the Formax has been an isolated body from its beginning, we must consider that a large-scale burst of star formation occurred as we observe today in M82, or else this Galaxy collided with intergalactic gas clouds with velocity more than $50-100 \mathrm{~km} / \mathrm{s}$.

The metallicity effects on the Cepheid distance scale Barry F. Madore, Wendy L. Freedman

There is a problem increasing the reddening of the LMC Cepheids to match the two Cloud $P C$ relations, the BVI reddening scale has been matched to Galactic Cepheids with known space reddenings, and with no reasonable metallicity can the LMC Cepheid reddenings be greatly increased.

1. Your reddenings in $M 31$ are heavily weighted by VRI which should have little metallicity dependence. In such a case the predicted deviation at $B$ is not $\sim 0.8 \mathrm{mag}$ but $\sim 0.813 \mathrm{mag}$. 
2. If indeed there is no metallicity dependence at $B$ the period $B-V$ relations will be the same in the Galaxy, LMC and SMC (one will need to adjust the reddening to get this). In that case, the period V-I relations, at present the same (as expected), will become different from galaxy to galaxy.

MADORE: $\quad$ 1. The prediction by Stothers is for the self-consistent redding - corrected true distance modullus. The M31 solutions using BVRI, BV CR VI only, all give consistent results, none of which confirms the large theoretical divergence. Within the errors the observations could accommodate a correction reduced by a factor of four.

2. We all intuitively expect some metallicity effect at B; the amplitude is now in question. At what level the coordinated adjustments of reddenings and samples will reveal a contradiction remains to be seen.

MOULD:

Wouldn't it be easier to compare theoretical predictions with bolometric magnitudes, which you can calculate since you have photometry from 0.4 to 2.2 microns?

MADORE:

Good idea. Still, one has to deal with the question of reddening, moreover, theory predicts a much reduced effect on bolometric magnitudes, the major effect being a differential one of line blanketing in combination with redistribution to which the bolometric luminosity is, by definition, insensitive.

Paper:

Author:

High-velocity HI near the Magellanic Clouds Ulrich Mebold

MEABURN:

HI clouds that collide with relative velocities of $100 \mathrm{~km} / \mathrm{s}$ will form radiative shock fronts on the collision interfaces. The usual range of optical emission lines should then be seen. i.e. $[O I I],[O I]$, etc. Have any been found?

MEBOLD: $\quad$ As yet, none. 